\title{
Using Monte Carlo method for simulation of electromagnetic radiation penetration in body's tissues
}

\author{
S. A. Mahdipour ${ }^{1}$, H. R. Abdi Roknabadi ${ }^{2}$, F. Ghalenovi ${ }^{1}$, H. Akbari Motlagh ${ }^{3}$ \\ ${ }^{1}$ Hakim Sabzevari University, Sabzevar, Iran \\ ${ }^{2}$ Yazd University, Yazd, Iran \\ ${ }^{3}$ Birjand University, Birjand, Iran
}

Article history:

E-mail: ali.mahdipour88@yahoo.com

Received September 2013

Accepted October 2013

Available online October 2013

\begin{abstract}
According to the physical nature of the penetration of electromagnetic radiation, in different body's tissues, this process can be simulated with using Monte-Carlo (based on random number generation) methods. In this study penetration of ray in the muscle and bony tissues is simulated. With using Fortran90 code, the penetration of electromagnetic ray in the cube target was simulated. The curve of ray penetration in the muscle and bony tissues, for different ray energies $(40,60,200,500 \mathrm{KeV})$ is obtained. So change the output ray intensity is calculated per number of different primary rays. This calculation compared with experimental calculations in water, with comparing the results of code, that written by authors and experimental results, the results show good agreement with each other.
\end{abstract}

Keywords: Monte Carlo, radiation, penetration.

\section{Introduction}

When electromagnetic radiations penetrate into the body, different tissues, depending on their density, absorb different amounts of radiations [1]. Electromagnetic radiation's penetration and their absorption in the tissue are like an exponential function which is shown by the following formula [2]:

$$
I=I_{0} e^{-\mu x}
$$

Where, $\mathrm{I}_{0}, \mathrm{I}, \mathrm{x}$, and $\mu$ are input ray intensity, intensity of transmitted ray, absorbent's thickness, and linear attenuation coefficient respectively. The linear attenuation coefficient is depended on incident ray energy and absorbent material. According to physical view, $\mu$ is the probability of ray interaction with the matter on the unit interval [3]. Usually the mass attenuation coefficient $\left(\mu_{m}\right)$ in the formula of linear attenuation coefficient:

$$
\mu=\mu_{m} \rho
$$


(Where $\rho$ is the density of matter) is expressed for different materials depending on the energies of incident ray in given tables.

Using random number generation method in recent years has found a wide application in physics [4]. Different simulation software's has been designed and used. The main problem of this software's is lack of access to software written codes. Monte Carlo method basis is using the process of random number generation which is applied to modulate the random-nature phenomena. Since the process of ray penetration to body tissues is random nature, it can be simulated based on Monte Carlo calculations.

The purpose of this paper is simulation of ray penetration process in the body's muscle and bone tissue by using Monte Carlo calculations, which has been simulated by writing computer code in FORTRAN programming language by the writers. Also, to evaluate the accuracy of this paper's calculations, to compare results of code calculation, experimental results [5] in the water phantom with linear attenuation coefficient $(0.315)$ is presented that indicates relatively good agreement between these results.

\section{Theory}

A model has been designed to stimulate ray penetration process to muscle and bone tissues of body. For this project, there are two nested cubes that the inner cube is bone tissue which is located within the muscle tissue. The dimensions of these cubes are $0.75 \mathrm{~cm} * 0.75 \mathrm{~cm} * 20 \mathrm{~cm}$ and $1 \mathrm{~cm} * 1 \mathrm{~cm} * 30 \mathrm{~cm}$ respectively. Y-Z plane has been considered as the vertical plane on direction of rays motions (figure 1 ). In line with the $X$-axis of the geometry, bone tissue will be started at the point $x=8$ and will be continued to the point $x=22$. Using the random nature generation process, (each generated number represents an incident ray to the considered target), we simulated the incident rays.

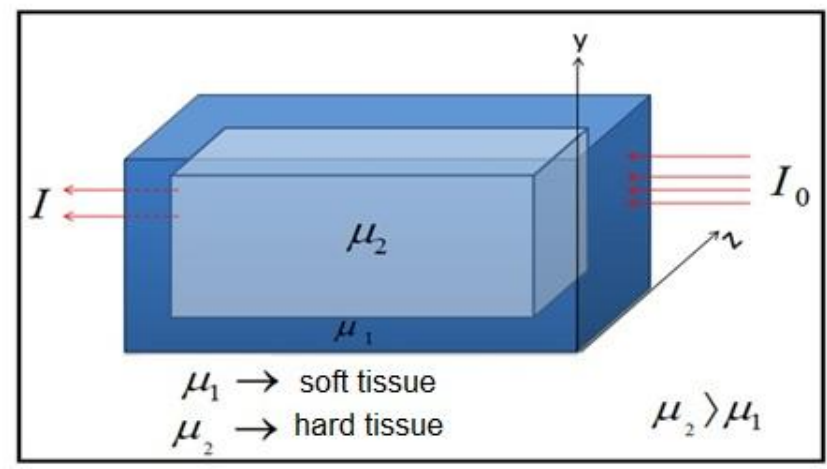

Figure 1. Geometry used in simulation process, soft tissue (muscle) and hard tissue (bone).

With looking to figure 1 , we see that some of rays, passing the hit target, just feel the muscle tissue and some others feel both tissues, that is to say muscle and bone tissue. By rays path division in the tissue to consecutive steps and according to the probability of these ray's interaction with soft and hard tissues $\left(\mu_{2}, \mu_{1}\right)$ (table 1 ), ray's numbers in each step has been calculated and incident ray intensity variation in thickness of $X$, has been plotted for different modes. To compare with experimental results, the inside target substance, water is considered with $\mu=0.315$ and ray penetration curve is calculated by running the code. 


\section{Results}

In figure 2, penetration curves of two different types of ray with the same energy of $500 \mathrm{KeV}$ which just one type encountered the muscle tissue through its moving path and the other type faced the muscle-bone tissue in its moving path is plotted. It's been observed that the absorption intensity of rays which must face bone tissue as well as muscle tissue through their path, while arriving to bone tissue zone $(x=8 \mathrm{~cm})$, increased and curve's slope in this zone $(x=8 \mathrm{~cm}$ up to $x=22 \mathrm{~cm})$ is associated with greater loss. This is because of the larger absorption coefficient of bone tissue in respect of absorption coefficient of muscle tissue [6].

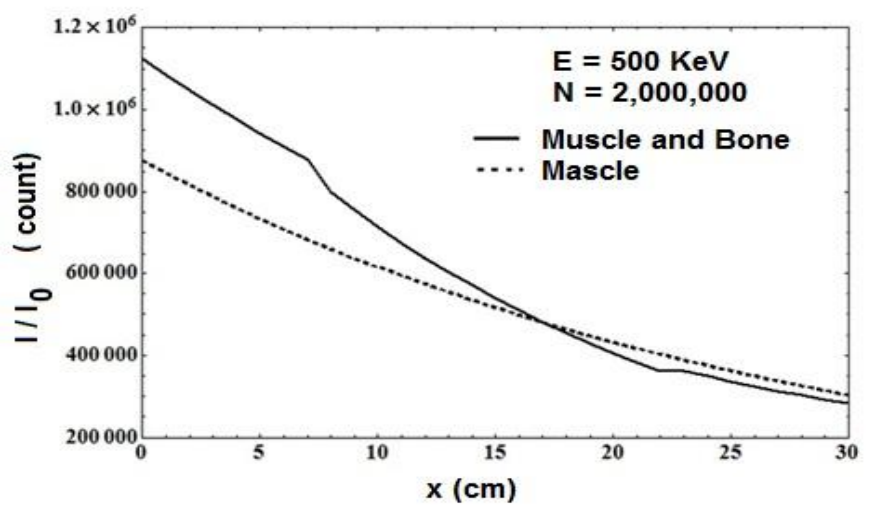

Figure 2. Penetration curve in muscle and bone tissue, for $500 \mathrm{KeV}$ energy and 2,000,000 primary rays.

Figure 3 shows the penetration ray curves in bone-muscle tissue for different energies of incident rays. According to this figure, rays of $40 \mathrm{KeV}$ energy at the depth about $15 \mathrm{~cm}$ (bone-tissue region) were absorbed completely. But rays of $60 \mathrm{keV}$ and $200 \mathrm{KeV}$ failed to pass the muscle and bone tissues, of course with different intensities. According to linear absorption coefficient with incident ray energy, the difference is justifiable [7].

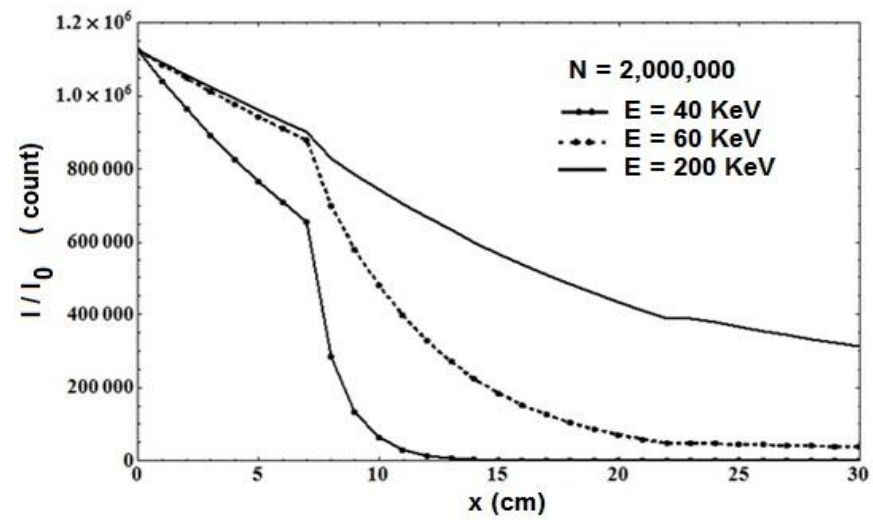

Figure 3. Penetration curve in muscle-bone tissue, for 2000,000 primary rays and energies of 40,60 , and $200 \mathrm{keV}$. 
Table 1. A calculated linear attenuation coefficient values for different energies [6].

\begin{tabular}{lcccc}
\hline Energy $(\mathrm{KeV})$ & Tissue & $\rho\left(\mathrm{g} / \mathrm{cm}^{3}\right)$ & $\mu_{\mathrm{m}}\left(\mathrm{cm}^{2} / \mathrm{g}\right)$ & $\mu\left(\mathrm{cm}^{-1}\right)$ \\
\hline $\mathbf{4 0}$ & Muscle & 1.059 & 0.0701 & 0.0742 \\
$\mathbf{4 0}$ & Bone & 1.750 & 0.304 & 0.532 \\
$\mathbf{6 0}$ & Muscle & 1.059 & 0.0328 & 0.0347 \\
$\mathbf{6 0}$ & Bone & 1.750 & 0.0998 & 0.174 \\
$\mathbf{2 0 0}$ & Muscle & 1.059 & 0.0294 & 0.0311 \\
$\mathbf{2 0 0}$ & Bone & 1.750 & 0.0301 & 0.0526 \\
$\mathbf{5 0 0}$ & Mascle & 1.059 & 0.0328 & 0.0347 \\
$\mathbf{5 0 0}$ & Bone & 1.750 & 0.0317 & 0.0554
\end{tabular}

Extreme changes of muscle-bone tissue output rays according to the numbers of primary rays in figure 4 have been reviewed. The more number of primary rays (random numbers) increases, the obtained values are closer to their actual values [8]. This is why that by increasing the number of primary rays, the curve's amplitude decreases and comes to actual value (almost about 3.3\%).

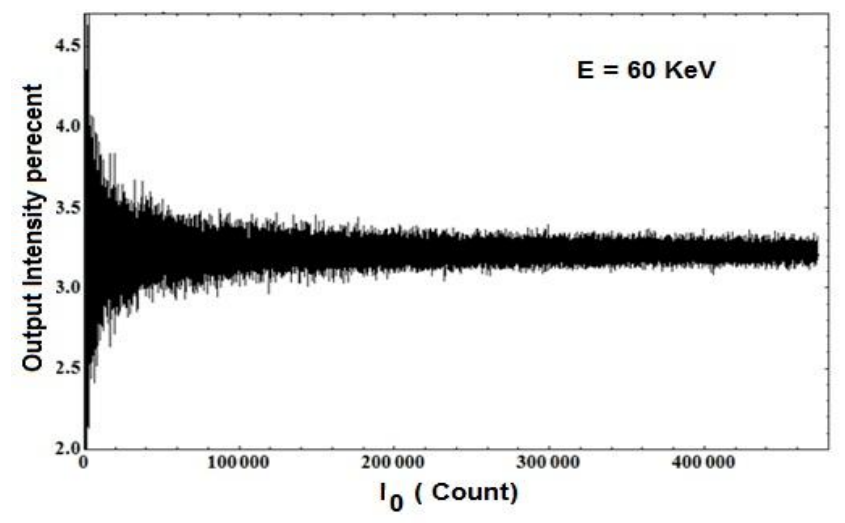

Figure 4. Intensity curve of muscle- bone tissue for different numbers of primary rays. 


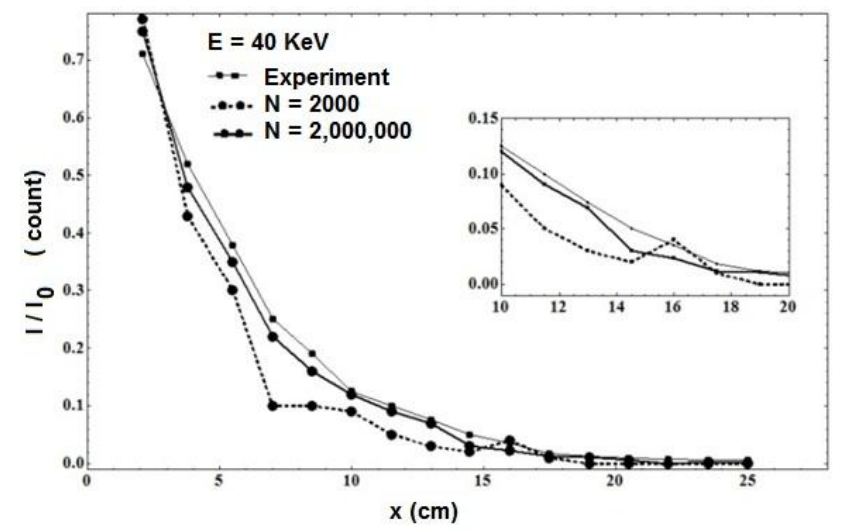

Figure 5. Monte Carlo and experimental curve of ray penetration into the water, for $40 \mathrm{KeV}$ energy [5].

In figure 5, comparison between the obtained curve of this code and experimental calculations curve, in a water phantom for $40 \mathrm{KeV}$ energy and $\mu=0.315$ [5], has been presented. To compare, curve's values have been calculated for one particle. According to this figure, the more generated points in simulation process (the number of primary rays), the less is the calculation errors and output values are closer to actual values. It's been observed that by changing the related linear attenuation coefficient of each tissue, the written code can stimulate the penetration ray in different body tissues based on Monte Carlo calculation with fairly reasonable accuracy. According to Monte Carlo - like simulation process and existed error in experimental conditions the difference between experimental and Monte Carlo results is justifiable.

According to obtained results, it's been observed that by increasing the number of primary rays in simulation process, calculation error decreases. But in turn the increase of primary rays numbers will increase the computation time by the code.

\section{Conclusions}

The penetration ray curve in body tissues has been calculated by the help of written code in FORTRAN 90 programming language based on Monte Carlo calculation which is generalizable to the materials. Also the comparison between this code's results with experimental results has a relatively proper accordance.

\section{References}

[1] James R. Connolly H Spring2012 EPS400 002

[2] J. Anthony Seibert, John M 2005 J. Nucl. Med. Technol. 331

[3] Weidner R. T, Sells R, Allyn and Bacon 1996 Elementary Modern Physics.

[4] B. Brown, William R. Martin, 1984 Progress in nuclear energy. 143 pp. 269-299

[5] CORRADO G, TESTOLIN, et al $2000 \mathrm{~J}$ Appl Physiol. 89 2365-2372

[6] Nicholas, Soulfanidis 1983 Measurement and detection of radiation.

[7] Pravina P. Pawar, 2011 Pharm. Res. 3(4):899-903

[8] William H. Press, et al 1993 Numerical Recipes in Fortran 